\title{
CONDITIONS FOR THE YONEDA ALGEBRA OF A LOCAL RING TO BE GENERATED IN LOW DEGREES
}

\author{
JUSTIN HOFFMEIER AND LIANA M. ŞEGA
}

\begin{abstract}
The powers $\mathfrak{m}^{n}$ of the maximal ideal $\mathfrak{m}$ of a local Noetherian ring $R$ are known to satisfy certain homological properties for large values of $n$. For example, the homomorphism $R \rightarrow R / \mathfrak{m}^{n}$ is Golod for $n \gg 0$. We study when such properties hold for small values of $n$, and we make connections with the structure of the Yoneda Ext algebra, and more precisely with the property that the Yoneda algebra of $R$ is generated in degrees 1 and 2. A complete treatment of these properties is pursued in the case of compressed Gorenstein local rings.
\end{abstract}

\section{INTRODUCTION}

Let $(R, \mathfrak{m}, k)$ be a local ring, that is, a commutative noetherian ring $R$ with unique maximal ideal $\mathfrak{m}$ and $k=R / \mathfrak{m}$. For $n \geq 1$ we let $\nu_{n}: \mathfrak{m}^{n} \rightarrow \mathfrak{m}^{n-1}$ denote the canonical inclusion and for each $i \geq 0$ we consider the induced maps

$$
\operatorname{Tor}_{i}^{R}\left(\nu_{n}, k\right): \operatorname{Tor}_{i}^{R}\left(\mathfrak{m}^{n}, k\right) \rightarrow \operatorname{Tor}_{i}^{R}\left(\mathfrak{m}^{n-1}, k\right) .
$$

Using the terminology of [2], we say that $\mathfrak{m}^{n}$ is a small submodule of $\mathfrak{m}^{n-1}$ if $\operatorname{Tor}_{i}^{R}\left(\nu_{n}, k\right)=0$ for all $i \geq 0$. This condition implies that the canonical projection $\rho_{n}: R \rightarrow R / \mathfrak{m}^{n}$ is a Golod homomorphism, but the converse may not hold.

Levin [9] showed that $\mathfrak{m}^{n}$ is a small submodule of $\mathfrak{m}^{n-1}$ for all sufficiently large values of $n$. On the other hand, the fact that $\mathfrak{m}^{n}$ is a small submodule of $\mathfrak{m}^{n-1}$ for small values of $n$ is an indicator of strong homological properties. It is known that $\mathfrak{m}^{2}$ is a small submodule of $\mathfrak{m}$ if and only if the Yoneda algebra $\operatorname{Ext}_{R}(k, k)$ is generated in degree 1, cf. [12, Corollary 1]. More generally, we show:

Theorem 1. Let $(R, \mathfrak{m}, k)$ be a local ring. Let $\widehat{R}=Q / I$ be a minimal Cohen presentation of $R$, with $(Q, \mathfrak{n}, k)$ a regular local ring and $I \subseteq \mathfrak{n}^{2}$. Let $t$ be an integer such that $I \subseteq \mathfrak{n}^{t}$. The following statements are then equivalent:

(1) $\mathfrak{m}^{t}$ is a small submodule of $\mathfrak{m}^{t-1}$;

(2) $\rho_{t}: R \rightarrow R / \mathfrak{m}^{t}$ is Golod;

(3) $\rho_{n}: R \rightarrow R / \mathfrak{m}^{n}$ is Golod for all $n$ such that $t \leq n \leq 2 t-2$;

(4) $I \cap \mathfrak{n}^{t+1} \subseteq \mathfrak{n} I$ and the algebra $\operatorname{Ext}_{R}^{*}(k, k)$ is generated by $\operatorname{Ext}_{R}^{1}(k, k)$ and $\operatorname{Ext}_{R}^{2}(k, k)$.

If $R$ is artinian, its socle degree is the largest integer $s$ with $\mathfrak{m}^{s} \neq 0$. When $R$ is a compressed Gorenstein local ring (see Section 3 for a definition) of socle degree $s \neq 3$, we determine all values of the integer $n$ for which the homomorphism

Research partly supported by NSF grant DMS-1101131 and grants from the Simons Foundation (\#20903 and \#354594, Liana Sega). 
$\rho_{n}$ is Golod, respectively for which $\mathfrak{m}^{n}$ is a small submodule of $\mathfrak{m}^{n-1}$, and we use Theorem 1 to establish part (3) below.

Theorem 2. Let $(R, \mathfrak{m}, k)$ be a compressed Gorenstein local ring of socle degree $s$. Assume $2 \leq s \neq 3$ and let $t$ denote the smallest integer such that $2 t \geq s+1$. If $n \geq 1$, then the following hold:

(1) $\mathfrak{m}^{n}$ is a small submodule of $\mathfrak{m}^{n-1}$ if and only if $n>s$ or $n=s+2-t$.

(2) $\rho_{n}: R \rightarrow R / \mathfrak{m}^{n}$ is Golod if and only if $n \geq s+2-t$.

(3) If $s$ is even, then $\operatorname{Ext}_{R}(k, k)$ is generated by $\operatorname{Ext}_{R}^{1}(k, k)$ and $\operatorname{Ext}_{R}^{2}(k, k)$.

The conclusion of (3) does not hold when $s$ is odd, see Corollary 3.7.

Section 1 provides definitions and properties of the homological notions of interest. Theorem 1 is proved in Section 2 and Theorem 2 is proved in Section 3.

\section{Preliminaries}

Throughout the paper $(R, \mathfrak{m}, k)$ denotes a commutative noetherian local ring with maximal ideal $\mathfrak{m}$ and residue field $k$. Let $M$ be a finitely generated $R$-module.

We denote by $\widehat{R}$ the completion of $R$ with respect to $\mathfrak{m}$. A minimal Cohen presentation of $R$ is a presentation $\widehat{R}=Q / I$, with $Q$ a regular local ring with maximal ideal $\mathfrak{n}$ and $I$ an ideal with $I \subseteq \mathfrak{n}^{2}$. We know that such a presentation exists, by the Cohen structure theorem.

We denote by $R^{\mathrm{g}}$ the associated graded ring with respect to $\mathfrak{m}$, and by $M^{\mathrm{g}}$ the associated graded module with respect to $\mathfrak{m}$. We denote by $\left(R^{\mathrm{g}}\right)_{j}$ the $j$-th graded component of $R^{\mathrm{g}}$. For any $x \in R$ we denote by $x^{*}$ the image of $x$ in $\mathfrak{m}^{j} / \mathfrak{m}^{j+1}=\left(R^{\mathrm{g}}\right)_{j}$, where $j$ is such that $x \in \mathfrak{m}^{j} \backslash \mathfrak{m}^{j+1}$. For an ideal $J$ of $R$, we denote by $J^{*}$ the homogeneous ideal generated by the elements $x^{*}$ with $x \in J$.

Remark 1.1. With $\widehat{R}=Q / I$ as above, the following then hold:

(1) $I \subseteq \mathfrak{n}^{t}$ if and only if $\operatorname{rank}_{k}\left(\mathfrak{m}^{t-1} / \mathfrak{m}^{t}\right)=\left(\begin{array}{c}e+t-2 \\ e-1\end{array}\right)$, where $e$ denotes the minimal number of generators of $\mathfrak{m}$.

(2) Assume $t \geq 2$ and $I \subseteq \mathfrak{n}^{t}$. Then $I \cap \mathfrak{n}^{t+1} \subseteq \mathfrak{n} I$ if and only if the map

$$
\operatorname{Ext}_{\rho_{t}}^{2}(k, k): \operatorname{Ext}_{R / \mathfrak{m}^{t}}^{2}(k, k) \rightarrow \operatorname{Ext}_{R}^{2}(k, k)
$$

induced by the canonical projection $\rho_{t}: R \rightarrow R / \mathfrak{m}^{t}$ is surjective.

To prove (1), note that $(\widehat{R})^{\mathrm{g}}=Q^{\mathrm{g}} / I^{*}$ and $Q^{\mathrm{g}}$ is isomorphic to a polynomial ring over $k$ in $e$ variables of degree 1 . We have that $I \subseteq \mathfrak{n}^{t}$ if and only if $I^{*} \subseteq\left(\mathfrak{n}^{\mathrm{g}}\right)^{t}$, which is equivalent to $\left(Q^{\mathrm{g}}\right)_{t-1}=\left(Q^{\mathrm{g}} / I^{*}\right)_{t-1}$ and thus to

$$
\operatorname{rank}_{k}\left(Q^{\mathrm{g}}\right)_{t-1}=\operatorname{rank}_{k}\left(\widehat{R}^{\mathrm{g}}\right)_{t-1} \text {. }
$$

Therefore, (1) follows by noting that $\operatorname{rank}_{k}\left(Q^{\mathrm{g}}\right)_{j}=\left(\begin{array}{c}e-1+j \\ e-1\end{array}\right)$ and $\operatorname{rank}_{k}\left(\widehat{R}^{\mathrm{g}}\right)_{j}=$ $\operatorname{rank}_{k}\left(\mathfrak{m}^{j} / \mathfrak{m}^{j+1}\right)$ for each $j$.

For a proof of $(2)$, see Şega $[15,4.3]$, noting that the map $\operatorname{Ext}_{\rho_{t}}^{2}(k, k)$ is surjective if and only if the induced map

$$
\operatorname{Tor}_{2}^{\rho_{t}}(k, k): \operatorname{Tor}_{2}^{R}(k, k) \rightarrow \operatorname{Tor}_{2}^{R / \mathfrak{m}^{t}}(k, k)
$$

is injective. 
Definition. Let $\widehat{R}=Q / I$ be a minimal Cohen presentation of $R$. Let $t \geq 2$ be an integer. We say that the local ring $R$ is $t$-homogeneous if $I \subseteq \mathfrak{n}^{t}$ and $I \cap \mathfrak{n}^{t+1} \subseteq \mathfrak{n} I$. Remark 1.1 shows that this definition does not depend on the choice of the minimal Cohen presentation.

We set

$$
v(R)=\sup \left\{t \geq 0 \mid I \subseteq \mathfrak{n}^{t}\right\} .
$$

Note that, if $R$ is $t$-homogeneous and $I \neq 0$, then $t=v(R)$.

Remark 1.2. The terminology of two-homogeneous algebra was previously used by Löfwall [11], with a different meaning, in the context of augmented graded algebras.

Lemma 1.3. Let $\widehat{R}=Q / I$ be a minimal Cohen presentation of $R$. If the ideal $I^{*}$ of the polynomial ring $Q^{\mathrm{g}}$ is generated by homogeneous polynomials of degree $t$, then the ring $R$ is t-homogeneous.

Proof. Assume $I^{*}$ is generated by homogeneous polynomials of degree $t$. In particular, it follows that $I \subseteq \mathfrak{n}^{t}$. To prove $I \cap \mathfrak{n}^{t+1} \subseteq \mathfrak{n} I$, we will show $I \cap \mathfrak{n}^{t+1} \subseteq \mathfrak{n} I+\mathfrak{n}^{j}$ for all $j \gg 0$. The Krull intersection theorem then gives the conclusion.

Let $x \in I \cap \mathfrak{n}^{t+1}$ and let $a \geq 1$ be the smallest integer such that $x \notin \mathfrak{n}^{t+1+a}$. Then $x^{*}$ is an element of degree $t+a$ of $I^{*}$. Since $I^{*}$ is generated by homogeneous elements of degree $t$, we can write

$$
x^{*}=\sum y_{i}^{*} z_{i}^{*}
$$

with $y_{i}^{*} \in\left(Q^{\mathrm{g}}\right)_{a}$ and $z_{i}^{*} \in I^{*} \cap\left(Q^{\mathrm{g}}\right)_{t}$ for each $i$, where $y_{i} \in \mathfrak{n}^{a}$ and $z_{i} \in I \cap \mathfrak{n}^{t}$. Set

$$
x_{1}=x-\sum y_{i} z_{i}
$$

and note that $x_{1} \in I \cap \mathfrak{n}^{t+a+1}$ and $x-x_{1} \in \mathfrak{n} I$. In particular $x \in \mathfrak{n} I+\mathfrak{n}^{t+a+1}$. Applying the argument above to $x_{1}$, we obtain an element $a_{1}$ such that $a_{1}>a$, and an element $x_{2}$ such that $x_{2} \in I \cap \mathfrak{n}^{t+a_{1}+1}$ and $x_{1}-x_{2} \in \mathfrak{n} I$. In particular $x_{1}$, and thus $x$, are elements of $\mathfrak{n} I+\mathfrak{n}^{t+a_{1}+1}$. An inductive argument produces a sequence of integers $1<a_{1}<a_{2}<\ldots$ such that $x \in \mathfrak{n} I+\mathfrak{n}^{t+a_{i}+1}$ for all $i$, and gives the desired conclusion.

Remark 1.4. The converse of the lemma does not hold. This can be seen by considering the 2-homogeneous local ring $R=k[[x, y]] /\left(x^{2}+y^{3}, x y\right)$, for which $R^{\mathrm{g}}=k[x, y] /\left(x^{2}, y^{4}, x y\right)$.

We now proceed to provide definitions for the homological notions of interest, and recall some of their properties.

The Poincaré series $\mathrm{P}_{M}^{R}(z)$ of $M$ is the formal power series

$$
\mathrm{P}_{M}^{R}(z)=\sum_{i \geq 0} \operatorname{rank}_{k}\left(\operatorname{Tor}_{i}^{R}(M, k)\right) z^{i} .
$$

1.5. Golod rings, modules, and homomorphisms. Let $(S, \mathfrak{s}, k)$ be a local ring and $\varphi: R \rightarrow S$ be a surjective homomorphism of local rings. Following Levin [10], we say that an $S$-module $M$ is $\varphi$-Golod if the following equality is satisfied:

$$
\mathrm{P}_{M}^{S}(z)=\frac{\mathrm{P}_{M}^{R}(z)}{\left(1-z\left(\mathrm{P}_{S}^{R}(z)-1\right)\right)} .
$$

We say that $\varphi$ is a Golod homomorphism if $k$ is a $\varphi$-Golod module. 
The ring $R$ is said to be a Golod ring if the canonical projection $Q \rightarrow \widehat{R}$ is a Golod homomorphism, where $\widehat{R}=Q / I$ is a minimal Cohen presentation. This definition is independent of the choice of representation by [1, Lemma 4.1.3]. Note that this definition is equivalent to the definition given by Avramov in terms of Koszul homology in $[1, \S 5]$. A classical example of a Golod ring is $Q / \mathfrak{n}^{j}$ for any $j \geq 2$, where $(Q, \mathfrak{n}, k)$ is a regular local ring, as first observed by Golod [5] .

1.6. Small homomorphisms. Let $\varphi: R \rightarrow S$ be a surjective ring homomorphism as above and consider the induced maps

$$
\begin{aligned}
& \operatorname{Ext}_{\varphi}^{i}(k, k): \operatorname{Ext}_{S}^{i}(k, k) \rightarrow \operatorname{Ext}_{R}^{i}(k, k) \\
& \operatorname{Tor}_{i}^{\varphi}(k, k): \operatorname{Tor}_{i}^{R}(k, k) \rightarrow \operatorname{Tor}_{i}^{S}(k, k) .
\end{aligned}
$$

We say that $\varphi$ is small if $\operatorname{Ext}_{\varphi}^{*}(k, k)$ is surjective or, equivalently, if $\operatorname{Tor}_{*}^{\varphi}(k, k)$ is injective. Note that $\operatorname{Tor}_{1}^{\varphi}(k, k)$ can be identified with the canonical map

$$
\mathfrak{m} / \mathfrak{m}^{2} \rightarrow \mathfrak{s} / \mathfrak{s}^{2}
$$

induced by $\varphi$. Thus $\operatorname{Tor}_{1}^{\varphi}(k, k)$ is an isomorphism if and only if $\operatorname{Ker}(\varphi) \subseteq \mathfrak{m}^{2}$.

For convenience we collect below a few known facts about small homomorphisms:

(1) If $\varphi$ is Golod then $\varphi$ is small, see Avramov [2, 3.5].

(2) If $S$ is a Golod ring and $\varphi$ is small, then $\varphi$ is Golod, see Şega [14, 6.7].

1.7. Inert modules. Let $\varkappa: P \rightarrow R$ be a surjective homomorphism of local rings. Following Lescot [7], we say that an $R$-module $M$ is inert by $\varkappa$ if the following equality holds:

$$
\mathrm{P}_{k}^{R}(z) \mathrm{P}_{M}^{P}(z)=\mathrm{P}_{k}^{P}(z) \mathrm{P}_{M}^{R}(z) .
$$

If $\varkappa$ is a Golod homomorphism the following are equivalent:

(1) $M$ is $\varkappa$-Golod;

(2) $\operatorname{Tor}_{i}^{\varkappa}(M, k)$ is injective for all $i$;

(3) $M$ is inert by $\varkappa$.

The equivalence of (1) and (3) is a direct consequence of the definitions and the equivalence of (1) with (2) is given by Levin [10, 1.1].

1.7.1. Consider a sequence of surjective homomorphisms of local rings

$$
R \stackrel{\alpha}{\rightarrow} S \stackrel{\beta}{\rightarrow} T \text {. }
$$

Lescot [7, Theorem 3.6] shows that a $T$-module $M$ is inert by $\beta \circ \alpha$ if an only if $M$ is inert by $\beta$ and $M$ is inert by $\alpha$, when considered as an $S$-module.

1.7.2. Let $Q$ be a regular local ring with maximal ideal $\mathfrak{n}$ and an ideal $J$ such that $J \subseteq \mathfrak{n}^{t}$ with $t \geq 2$. Set $S=Q / J$ and $\overline{\mathfrak{n}}=\mathfrak{n} / J$. If a finitely generated $S$-module $N$ is annihilated by $\overline{\mathfrak{n}}^{t-1}$ then $N$ is inert by the natural projection $\varkappa: Q \rightarrow S$, see $[7$, 3.7 and 3.11]

We end this section with introducing some more notation.

1.8. If $(R, \mathfrak{m}, k)$ is a local ring and $M$ is a finite $R$-module, we let $\nu_{M}: \mathfrak{m} M \rightarrow M$ denote the canonical inclusion and consider the induced maps

$$
\operatorname{Tor}_{i}^{R}\left(\nu_{M}, k\right): \operatorname{Tor}_{i}^{R}(\mathfrak{m} M, k) \rightarrow \operatorname{Tor}_{i}^{R}(M, k) .
$$

They fit into the long exact sequence

$$
\cdots \rightarrow \operatorname{Tor}_{i}^{R}(\mathfrak{m} M, k) \stackrel{\operatorname{Tor}_{i}^{R}\left(\nu_{M}, k\right)}{\longrightarrow} \operatorname{Tor}_{i}^{R}(M, k) \rightarrow \operatorname{Tor}_{i}^{R}(M / \mathfrak{m} M, k) \rightarrow \cdots .
$$


If $\Lambda$ is a graded vector space over $k$, we set $H_{\Lambda}(z)=\sum_{i>0} \operatorname{rank}_{k}\left(\Lambda_{i}\right) z^{i}$; this formal power series is called the Hilbert series of $\Lambda$. Since rank is additive on exact sequences, a rank count in the exact sequence above gives

$$
\mathrm{P}_{M}^{R}(z)-a \mathrm{P}_{k}^{R}(z)+z \mathrm{P}_{\mathfrak{m} M}^{R}(z)=(1+z) H_{\operatorname{Im}\left(\operatorname{Tor}_{*}^{R}\left(\nu_{M}, k\right)\right)}(z)
$$

where $a=\operatorname{rank}_{k}(M / \mathfrak{m} M)$. We set

$$
T_{M}^{R}(z)=H_{\operatorname{Im}\left(\operatorname{Tor}_{*}^{R}\left(\nu_{M}, k\right)\right)}(z) .
$$

2. Homological properties of powers of the MaXimal ideal

The purpose of this section is to prove Theorem 1 in the introduction, restated as Theorem 2.5 below.

For each integer $j$ let

$$
\rho_{j}: R \rightarrow R / \mathfrak{m}^{j} \quad \text { and } \quad \nu_{j}: \mathfrak{m}^{j} \rightarrow \mathfrak{m}^{j-1}
$$

denote the canonical projection, respectively the inclusion, and consider the induced maps

$$
\begin{gathered}
\operatorname{Tor}_{i}^{\rho_{j}}\left(R / \mathfrak{m}^{j-1}, k\right): \operatorname{Tor}_{i}^{R}\left(R / \mathfrak{m}^{j-1}, k\right) \rightarrow \operatorname{Tor}_{i}^{R / \mathfrak{m}^{j}}\left(R / \mathfrak{m}^{j-1}, k\right) \\
\operatorname{Tor}_{i}^{R}\left(\nu_{j}, k\right): \operatorname{Tor}_{i}^{R}\left(\mathfrak{m}^{j}, k\right) \rightarrow \operatorname{Tor}_{i}^{R}\left(\mathfrak{m}^{j-1}, k\right) .
\end{gathered}
$$

Using the terminolgy in the appendix of [2], we say that $\mathfrak{m}^{j}$ is a small submodule of $\mathfrak{m}^{j-1}$ if $\operatorname{Tor}_{i}^{R}\left(\nu_{j}, k\right)=0$ for all $i \geq 0$.

Remark 2.1. For $i, j \geq 0$ let

$$
\eta_{i}^{j}: \operatorname{Tor}_{i}^{R}\left(R / \mathfrak{m}^{j}, k\right) \rightarrow \operatorname{Tor}_{i}^{R}\left(R / \mathfrak{m}^{j-1}, k\right)
$$

denote the map induced by the canonical projection $R / \mathfrak{m}^{j} \rightarrow R / \mathfrak{m}^{j-1}$.

Note that $\operatorname{Tor}_{i}^{R}\left(\nu_{j}, k\right)=0$ if and only if $\eta_{i+1}^{j}=0$. Indeed, this is a standard argument, using the canonical isomorphisms $\operatorname{Tor}_{i+1}^{R}\left(R / \mathfrak{m}^{n}, k\right) \cong \operatorname{Tor}_{i}^{R}\left(\mathfrak{m}^{n}, k\right)$ which arise as connecting homomorphisms in the long exact sequence associated to the exact sequence

$$
0 \rightarrow \mathfrak{m}^{n} \rightarrow R \rightarrow R / \mathfrak{m}^{n} \rightarrow 0,
$$

with $n=j$ and $n=j-1$.

2.2. We state here a needed result of Rossi and Şega [13, Lemma 1.2]:

Let $\varkappa:(P, \mathfrak{p}, k) \rightarrow(R, \mathfrak{m}, k)$ be a surjective homomorphism of local rings. Assume there exists an integer $a$ such that

(1) The map $\operatorname{Tor}_{i}^{P}(R, k) \rightarrow \operatorname{Tor}_{i}^{P}\left(R / \mathfrak{m}^{a}, k\right)$ induced by the natural projection $R \rightarrow R / \mathfrak{m}^{a}$ is zero for all $i>0$.

(2) The map $\operatorname{Tor}_{i}^{P}\left(\mathfrak{m}^{2 a}, k\right) \rightarrow \operatorname{Tor}_{i}^{P}\left(\mathfrak{m}^{a}, k\right)$ induced by the inclusion $\mathfrak{m}^{2 a} \hookrightarrow \mathfrak{m}^{a}$ is zero for all $i \geq 0$.

Then $\varkappa$ is a Golod homomorphism.

Proposition 2.3. Let $(R, \mathfrak{m}, k)$ be a local ring and let $j \geq 2$ be an integer. The following are equivalent:

(1) $\mathfrak{m}^{j}$ is a small submodule of $\mathfrak{m}^{j-1}$;

(2) $\operatorname{Tor}_{i}^{\rho_{j}}\left(R / \mathfrak{m}^{j-1}, k\right)$ is injective for all $i \geq 0$;

(3) $\rho_{j}$ is Golod and $R / \mathfrak{m}^{j-1}$ is inert by $\rho_{j}$.

If these conditions hold, then $\rho_{l}$ is Golod for all integers $l$ with $j \leq l \leq 2 j-2$. 
Proof. $(1) \Rightarrow(2)$ : Let $i \geq 0$. Set $\overline{\mathfrak{m}}^{j-1}=\mathfrak{m}^{j-1} / \mathfrak{m}^{j}$. Consider long exact sequences associated to the exact sequence

$$
0 \rightarrow \overline{\mathfrak{m}}^{j-1} \rightarrow R / \mathfrak{m}^{j} \rightarrow R / \mathfrak{m}^{j-1} \rightarrow 0
$$

and create the following commutative diagram with exact columns.

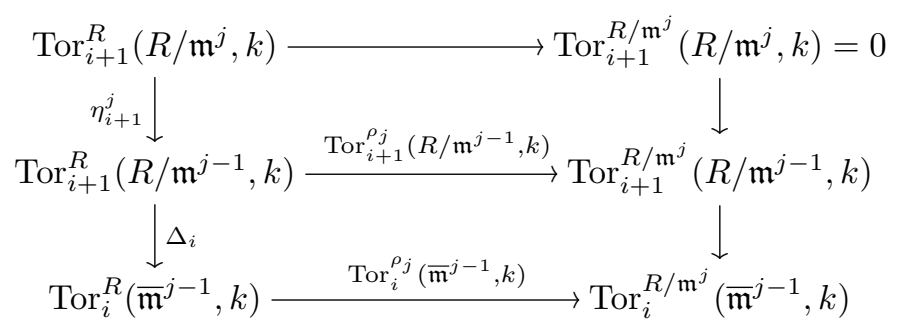

By Remark 2.1, the hypothesis that $\operatorname{Tor}_{i}^{R}\left(\nu_{j}, k\right)=0$ implies that $\eta_{i+1}^{j}=0$, and thus the connecting homomorphism $\Delta_{i}$ is injective.

Levin's proof of $[9,3.15]$ shows $\operatorname{Tor}_{i}^{R}\left(\nu_{j}, k\right)=0$ for all $i$ implies $\rho_{j}$ is Golod. (This also follows from the last part of the proof.) In particular, the map $\rho_{j}$ is small by 1.6(1). Since $\overline{\mathfrak{m}}^{j-1}$ is a direct sum of copies of $k$, it follows that $\operatorname{Tor}_{i}^{\rho_{j}}\left(\overline{\mathfrak{m}}^{j-1}, k\right)$ is injective.

The bottom commutative square yields that $\operatorname{Tor}_{i+1}^{\rho_{j}}\left(R / \mathfrak{m}^{j-1}, k\right)$ is injective.

$(2) \Rightarrow(1)$ : Assuming that $\operatorname{Tor}_{i+1}^{\rho_{j}}\left(R / \mathfrak{m}^{j-1}, k\right)$ is injective, the top square in the commutative diagram above gives that $\eta_{i+1}^{j}=0$, and thus $\operatorname{Tor}_{i}^{R}\left(\nu_{j}, k\right)=0$ by Remark 2.1.

$(1) \Rightarrow(3)$ : As mentioned above, $\operatorname{Tor}_{i}^{R}\left(\nu_{j}, k\right)=0$ for all $i$ implies that $\rho_{j}$ is Golod. Since we already proved $(1) \Rightarrow(2)$, we know that $\operatorname{Tor}_{i}^{\rho_{j}}\left(R / \mathfrak{m}^{j-1}, k\right)$ is injective for all $i$. By $1.7, R / \mathfrak{m}^{j-1}$ is then inert by $\rho_{j}$.

$(3) \Rightarrow(2)$ : see 1.7 .

Fix now $l$ such that $j \leq l \leq 2 j-2$. We prove the last assertion of the proposition by applying 2.2 , with $\varkappa=\rho_{l}$ and $a=j-1$. Set $\bar{R}=R / \mathfrak{m}^{l}$ and $\overline{\mathfrak{m}}=\mathfrak{m} / \mathfrak{m}^{l}$. Let

$$
\bar{\rho}_{j-1}: \bar{R} \rightarrow \bar{R} / \overline{\mathfrak{m}}^{j-1}
$$

denote the canonical projection. To satisfy the first hypothesis of 2.2 , we will show that the induced map

$$
\operatorname{Tor}_{i}^{R}\left(\bar{\rho}_{j-1}, k\right): \operatorname{Tor}_{i}^{R}(\bar{R}, k) \rightarrow \operatorname{Tor}_{i}^{R}\left(\bar{R} / \overline{\mathfrak{m}}^{j-1}, k\right)
$$

is zero for all $i>0$. Since $l \geq j$, we have $\bar{R} / \overline{\mathfrak{m}}^{j-1}=R / \mathfrak{m}^{j-1}$ and $\operatorname{Tor}_{i}^{R}\left(\bar{\rho}_{j-1}, k\right)$ factors through

$$
\eta_{i}^{j}: \operatorname{Tor}_{i}^{R}\left(R / \mathfrak{m}^{j}, k\right) \rightarrow \operatorname{Tor}_{i}^{R}\left(R / \mathfrak{m}^{j-1}, k\right) .
$$

Since $\operatorname{Tor}_{i}^{R}\left(\nu_{j}, k\right)=0$ for all $i \geq 0$ by assumption, we have that $\eta_{i}^{j}=0$ for all $i>0$ by Remark 2.1. Hence $\operatorname{Tor}_{i}^{R}\left(\bar{\rho}_{j-1}, k\right)=0$ for all $i>0$.

To satisfy the second hypothesis of 2.2 , we need to show that the induced map

$$
\operatorname{Tor}_{i}^{R}\left(\overline{\mathfrak{m}}^{2(j-1)}, k\right) \rightarrow \operatorname{Tor}_{i}^{R}\left(\overline{\mathfrak{m}}^{j-1}, k\right)
$$

induced by the inclusion $\overline{\mathfrak{m}}^{2(j-1)} \hookrightarrow \overline{\mathfrak{m}}^{j-1}$ is zero for all $i \geq 0$. In fact, this map is trivially zero since the inclusion $\mathfrak{m}^{2 j-2} \subseteq \mathfrak{m}^{l}$ (given by the inequality $l \leq 2 j-2$ ) implies $\overline{\mathfrak{m}}^{2 j-2}=0$. Hence $\rho_{l}$ is Golod by 2.2 . 
Lemma 2.4. Let $(R, \mathfrak{m}, k)$ be a local ring. If an integer $t$ satisfies $2 \leq t \leq v(R)$, then $R / \mathfrak{m}^{t-1}$ is inert by $\rho_{t}$.

Proof. We may assume that $R$ is complete. Let $R=Q / I$ be a minimal Cohen presentation, with $(Q, \mathfrak{n}, k)$ a regular local ring. Since $t \leq v(R)$, we have $I \subseteq \mathfrak{n}^{t}$. We can make thus the identification $R / \mathfrak{m}^{j}=Q / \mathfrak{n}^{j}$ for all $j \leq t$. Let $\varkappa: Q \rightarrow R$ and $\alpha_{t}: Q \rightarrow Q / \mathfrak{n}^{t}$ denote the canonical projections. Since $\alpha_{t}=\rho_{t} \circ \varkappa, 1.7 .1$ shows that it suffices to prove that $R / \mathfrak{m}^{t-1}$ is inert by $\alpha_{t}$. This can be seen by applying 1.7.2 with $J=\mathfrak{n}^{t}, S=Q / \mathfrak{n}^{t}, \overline{\mathfrak{n}}=\mathfrak{n} / \mathfrak{n}^{t}$ and $M=S / \overline{\mathfrak{n}}^{t-1}=Q / \mathfrak{n}^{t-1}$.

Theorem 2.5. Let $(R, \mathfrak{m}, k)$ be a local ring and let $t$ be an integer satisfying $2 \leq$ $t \leq v(R)$. The following are equivalent:

(1) $\mathfrak{m}^{t}$ is a small submodule of $\mathfrak{m}^{t-1}$;

(2) $\rho_{t}$ is small;

(3) $\rho_{j}$ is small for all $j \geq t$;

(4) $\rho_{t}$ is Golod;

(5) $\rho_{j}$ is Golod for all $j$ such that $t \leq j \leq 2 t-2$;

(6) $R$ is t-homogeneous and the algebra $\operatorname{Ext}_{R}^{*}(k, k)$ is generated by $\operatorname{Ext}_{R}^{1}(k, k)$ and $\operatorname{Ext}_{R}^{2}(k, k)$.

Proof. The homological properties under consideration are invariant under completion. We may assume thus $R$ is complete. Hence $R=Q / I$ with $(Q, \mathfrak{n}, k)$ a regular local ring and $I \subseteq \mathfrak{n}^{t}$, with $t \geq 2$. In particular, we can make the identification $R / \mathfrak{m}^{t}=Q / \mathfrak{n}^{t}$.

$(2) \Rightarrow(3)$ : This follows immediately from the definition of small homomorphim.

$(3) \Rightarrow(2)$ : Clear.

$(3) \Rightarrow(4)$ : Since $R / \mathfrak{m}^{t}=Q / \mathfrak{n}^{t}$ is Golod (see 1.5), we can apply 1.6(2).

$(4) \Rightarrow(2)$ : See 1.6(1).

$(4) \Rightarrow(1)$ : By assumption $\rho_{t}$ is Golod. By Lemma $2.4, R / \mathfrak{m}^{t-1}$ is inert by $\rho_{t}$. Hence $\mathfrak{m}^{t}$ is a small submodule of $\mathfrak{m}^{t-1}$ by Proposition 2.3 .

$(1) \Rightarrow(5)$ : See Proposition 2.3 .

$(5) \Rightarrow(4)$ : Clear.

$(2) \Rightarrow(6)$ : Assume $\rho_{t}$ is small, hence $\operatorname{Ext}_{\rho_{t}}(k, k)$ is a surjective homomorphism of graded algebras. In $[8,5.9]$, Levin shows $\operatorname{Ext}_{Q / \mathfrak{n}^{t}}(k, k)$ is generated by elements in degree 1 and 2. It follows that $\operatorname{Ext}_{R}(k, k)$ is also generated in degrees 1 and 2. To see that $R$ is $t$-homogeneous, use Remark 1.1(2).

$(6) \Rightarrow(2)$ : Assume $R$ is $t$-homogeneous and the Yoneda algebra $\operatorname{Ext}_{R}(k, k)$ is generated by $\operatorname{Ext}_{R}^{1}(k, k)$ and $\operatorname{Ext}_{R}^{2}(k, k)$. To show that $\operatorname{Ext}_{\rho_{t}}(k, k)$ is surjective, it suffices to show that $\operatorname{Ext}_{\rho_{t}}^{1}(k, k)$ and $\operatorname{Ext}_{\rho_{t}}^{2}(k, k)$ are surjective. Since $t \geq 2$, we have that $\operatorname{Ker}\left(\rho_{t}\right) \subseteq \mathfrak{m}^{2}$, hence $\operatorname{Ext}_{\rho_{t}}^{1}(k, k)$ is an isomorphism, as discussed in 1.6. The fact that $\operatorname{Ext}_{\rho_{t}}^{2}(k, k)$ is surjective is given by Remark 1.1(2).

We say that $R$ is a complete intersection if the ideal $I$ in a minimal Cohen presentation $\widehat{R}=Q / I$ is generated by a regular sequence. For such rings, the structure of the algebra $\operatorname{Ext}_{R}(k, k)$ is known, see Sjödin [16, §4]. In particular, it is known that this algebra is generated in degrees 1 and 2 .

Corollary 2.6. If $R$ is a t-homogeneous complete intersection, then conditions (1)-(5) of the Theorem hold. 
Remark 2.7. Connected $k$-algebras satisfying the condition that the Yoneda algebra is generated in degrees 1 and 2 are called $\mathcal{K}_{2}$ algebras by Cassidy and Shelton [4]. Since Koszul algebras are characterized by the fact that their Yoneda algebras are generated in degree 1 , the notion of $\mathcal{K}_{2}$ algebra can be thought of as a generalization of the notion of Koszul algebra.

\section{Compressed Gorenstein LOCAL Rings}

Compressed Gorenstein local rings have been recently studied by Rossi and Şega [13]; we recall below the definition given there. We consider this large class of rings as a case study for the homological properties of interest.

3.1. Compressed Gorenstein local rings. Let $(R, \mathfrak{m}, k)$ be a Gorenstein artinian local ring. The embedding dimension of $R$ is the integer $e=\operatorname{rank}_{k}\left(\mathfrak{m} / \mathfrak{m}^{2}\right)$, and the socle degree of $R$ is the integer $s$ such that $\mathfrak{m}^{s} \neq 0=\mathfrak{m}^{s+1}$. Since $R$ is complete, a minimal Cohen presentation of $R$ is $R=Q / I$ with $(Q, \mathfrak{n}, k)$ a regular local ring and $I \subseteq \mathfrak{n}^{2}$. Set

$$
\varepsilon_{i}=\min \left\{\left(\begin{array}{c}
e-1+s-i \\
e-1
\end{array}\right),\left(\begin{array}{c}
e-1+i \\
e-1
\end{array}\right)\right\} \quad \text { for all } i \text { with } 0 \leq i \leq s .
$$

According to $[13,4.2]$, we have

$$
\lambda(R) \leq \sum_{i=0}^{e} \varepsilon_{i}
$$

where $\lambda(R)$ denotes the length of $R$. We say that $R$ is a compressed Gorenstein local ring of socle degree $s$ and embedding dimension $e$ if $R$ has maximal length, that is, equality holds in (3.1.1).

If $R$ as above is compressed, we set

$$
t=\left\lceil\frac{s+1}{2}\right\rceil \text { and } r=s+1-t,
$$

where $\lceil x\rceil$ denotes the smallest integer not less than a rational number $x$.

As discussed in $[13,4.2]$, we have $t=v(R)$. Note that if $s$ is even then $s=2 t-2$ and $r=t-1$. If $s$ is odd then $s=2 t-1$ and $r=t$.

Remark 3.2. It is shown in $[13,4.2(\mathrm{c})]$ that if $R$ is a compressed Gorenstein local ring, then $R^{\mathrm{g}}$ is Gorenstein, and it is thus a compressed Gorenstein $k$-algebra. Note that compressed Gorenstein algebras can be regarded as being generic Gorenstein algebras, see the discussion in $[13,5.5]$.

Let $R$ be a compressed Gorenstein local ring of socle degree $s$. When $s$ is even, the minimal free resolution of $R^{\mathrm{g}}$ over $Q^{\mathrm{g}}$ is described for example by Iarrobino in $[6,4.7]$; in particular, it follows that $I^{*}$ is generated by homogeneous polynomials of degree $t$. According to Lemma 1.3, it follows that $R$ is $t$-homogeneous.

When $s$ is odd, $I^{*}$ can be generated in degrees $t$ and $t+1$; see [3, Proposition $3.2]$. It is conjectured in $[3,3.13]$ that $I^{*}$ is generated in degree $t$, and thus it is $t$-homogeneous, when $R^{\mathrm{g}}$ is generic in a stronger sense.

For the remainder of the section we use the assumptions and notation below.

3.3. Let $(R, \mathfrak{m}, k)$ be a compressed Gorenstein local ring of embedding dimension $e$ and socle degree $s$, with $2 \leq s \neq 3$. We consider a minimal Cohen presentation $R=Q / I$ with $(Q, \mathfrak{n}, k)$ a regular local ring and $I \subseteq \mathfrak{n}^{2}$. Since $t=v(R)$ we have 
$I \subseteq \mathfrak{n}^{t}$ and $I \nsubseteq \mathfrak{n}^{t+1}$. Let $h \in I \cap \mathfrak{n}^{t} \backslash \mathfrak{n}^{t+1}$. Set $P=Q /(h)$ and $\mathfrak{p}=\mathfrak{n} /(h)$. Let $\varkappa: P \rightarrow R$ denote the canonical projection. The following properties shown in [13] will be useful for our approach:

(1) $\mathfrak{m}^{r+1}$ is a small submodule of $\mathfrak{m}^{r}([13$, Theorem 3.3]);

(2) $R / \mathfrak{m}^{j}$ is a Golod ring for $2 \leq j \leq s$ ([13, Proposition 6.3]);

(3) $\varkappa: P \rightarrow R$ is a Golod homomorphism ([13, Theorem 5.1]).

(4) $\mathrm{P}_{k}^{R}(z) \cdot d_{R}(z)=\mathrm{P}_{k}^{Q}(z)$ (see [13, Theorem 5.1]), where

$$
d_{R}(z)=1-z\left(\mathrm{P}_{R}^{Q}(z)-1\right)+z^{e+1}(1+z) .
$$

Note that $d_{R}(z)$ is polynomial of degree $e+2$, since $\mathrm{P}_{R}^{Q}(z)$ is a polynomial of degree $e$.

Remark 3.4. Let $\eta: Q \rightarrow P$ denote the canonical projection. If $M$ is an $R$-module with $\mathfrak{m}^{t-1} M=0$, then 1.7.2 shows that $M$ is inert by $\varkappa \circ \eta$, since $I \subseteq \mathfrak{n}^{t}$. It follows that $M$ is also inert by $\varkappa$, by 1.7 .1 .

Note that the condition $\mathfrak{m}^{t-1} M=0$ is satisfied for $M=R / \mathfrak{m}^{j}$ with $j \leq t-1$ and also for $M=\mathfrak{m}^{j}$ with $j \geq r+1$ (since $t-1+r+1=s+1$ ), and thus $M$ is inert by $\varkappa$ and by $\varkappa \circ \eta$, by the above. The case $M=\mathfrak{m}^{r}$ is treated below.

Lemma 3.5. The R-module $\mathfrak{m}^{r}$ is inert by $\varkappa$.

Proof. Let $i \geq 0$. Consider the commutative diagram:

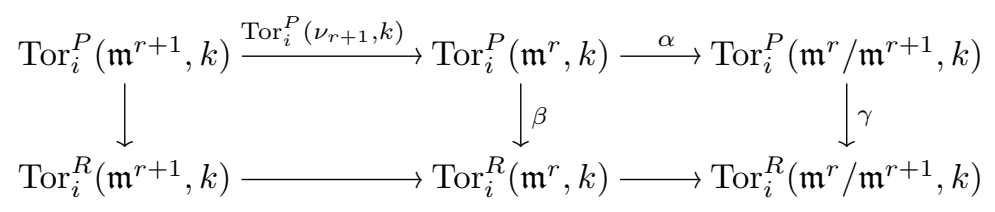

where $\beta=\operatorname{Tor}_{i}^{\varkappa}\left(\mathfrak{m}^{r}, k\right)$ and $\gamma=\operatorname{Tor}_{i}^{\varkappa}\left(\mathfrak{m}^{r} / \mathfrak{m}^{r+1}, k\right)$. Since $\operatorname{Tor}_{i}^{P}\left(\nu_{r+1}, k\right)=0$ by $3.3(1), \alpha$ is injective. Since $\varkappa$ is Golod, it is in particular small by 1.6(1), and it follows that $\gamma$ is injective, since $\mathfrak{m}^{r} / \mathfrak{m}^{r+1}$ is a direct sum of copies of $k$. The commutative square on the right shows that $\beta$ is injective as well, hence $\mathfrak{m}^{r}$ is inert by $\varkappa$ by 1.7 .

We now prove Theorem 2 in the introduction. We restate it below, with some more detail in part (1).

Theorem 3.6. Let $2 \leq s \neq 3$ and let $R$ be a compressed Gorenstein local ring of socle degree s. Let $n \geq 1$. The following hold:

(1) $\mathfrak{m}^{n}$ is a small submodule of $\mathfrak{m}^{n-1}$ if and only if $n>s$ or $n=r+1$. Furthermore, if $n \neq r+1$ and $n \leq s$, then $\operatorname{Tor}_{i}^{R}\left(\nu_{n}, k\right) \neq 0$ for infinitely many values of $i$.

(2) $\rho_{n}: R \rightarrow R / \mathfrak{m}^{n}$ is Golod if and only if $n \geq r+1$.

Corollary 3.7. With $R$ as in the theorem, the following hold:

(1) If $s$ is even, then $\operatorname{Ext}_{R}(k, k)$ is generated by $\operatorname{Ext}_{R}^{1}(k, k)$ and $\operatorname{Ext}_{R}^{2}(k, k)$.

(2) If $s$ is odd and $R$ is t-homogeneous, then $\operatorname{Ext}_{R}(k, k)$ is not generated by $\operatorname{Ext}_{R}^{1}(k, k)$ and $\operatorname{Ext}_{R}^{2}(k, k)$.

Proof. If $s$ is even, then $r=t-1$ and Theorem 3.6(1) gives that $\mathfrak{m}^{t}$ is a small submodule of $\mathfrak{m}^{t-1}$. If $s$ is odd, then $r=t$, and Theorem 3.6(1) gives that 
$\operatorname{Tor}_{i}^{R}\left(\nu_{t}, k\right) \neq 0$ for infinitely many values of $i$, hence $\mathfrak{m}^{t}$ is not a small submodule of $\mathfrak{m}^{t-1}$. Both conclusions follow then from Theorem 2.5.

Proof of Theorem 3.6. Assuming that (1) is proved, we prove (2) as follows.

Since $\mathfrak{m}^{r+1}$ is a small submodule of $\mathfrak{m}^{r}$, we know that that $\rho_{r+1}$ is a Golod homomorphism, and furthermore a small homomorphism (see Section 2). Let $n \geq$ $r+1$. The homomorphism $\rho_{n}$ factors thorugh $\rho_{r+1}$, and it is thus small as well. Since $R / \mathfrak{m}^{j}$ is Golod by 3.3(2), it follows by (2) in 1.6 that $\rho_{j}$ is Golod. If $n \leq r$, then we also have $n \leq t$, since $r=t$ or $r=t-1$. In view of Theorem 2.5 , the fact that $\operatorname{Tor}_{*}^{R}\left(\nu_{n}, k\right) \neq 0$ in this case implies that $\rho_{n}$ is not Golod.

We now prove (1). Let $j \geq 0$. We use the notation introduced in 1.8 , noting that $\nu_{\mathfrak{m}^{j}}=\nu_{j+1}$. We have

$$
\operatorname{Tor}_{*}^{R}\left(\nu_{j+1}, k\right)=0 \Longleftrightarrow T_{\mathfrak{m}^{j}}^{R}(z)=0
$$

and $\operatorname{Tor}_{i}^{R}\left(\nu_{j+1}, k\right) \neq 0$ for infinitely many $i$ if and only if $T_{\mathfrak{m}^{j}}^{R}(z) \notin \mathbb{Z}[z]$.

The conclusion will be established through a concrete computation of $T_{\mathfrak{m}^{j}}^{R}(z)$.

Using (1.8.1) we have:

$$
\mathrm{P}_{\mathfrak{m}^{j}}^{S}(z)-a_{j} \mathrm{P}_{k}^{S}(z)+z \mathrm{P}_{\mathfrak{m}^{j+1}}^{S}(z)=(1+z) T_{\mathfrak{m}^{j}}^{S}(z)
$$

where $a_{j}=\operatorname{rank}_{k}\left(\mathfrak{m}^{j} / \mathfrak{m}^{j+1}\right)$ and $S=R$ or $S=P$ or $S=Q$. There are four distinct cases to be considered:

Case 1. Assume $j=r$. Recall that $\mathfrak{m}^{r+1}$ and $\mathfrak{m}^{r}$ and $k$ are all inert by $\varkappa$, by 3.5 and 3.4. Using the definition of inertness for each of these modules, an application of the formula (3.7.1) for $j=r$, with $S=R$ and then with $S=P$, gives:

$$
T_{\mathfrak{m}^{r}}^{R}(z)=T_{\mathfrak{m}^{r}}^{P}(z) \cdot \frac{\mathrm{P}_{k}^{R}(z)}{\mathrm{P}_{k}^{P}(z)} .
$$

Since we know that $\operatorname{Tor}_{*}^{P}\left(\nu_{r+1}, k\right)=0$, see $3.3(1)$, we have that $T_{\mathfrak{m}^{r}}^{P}(z)=0$, hence $T_{\mathfrak{m}^{r}}^{R}(z)=0$ and thus $\operatorname{Tor}_{*}^{R}\left(\nu_{r+1}, k\right)=0$.

Case 2. Assume $r+1 \leq j<s$. We know that $\mathfrak{m}^{j+1}, \mathfrak{m}^{j}$ and $k$ are all inert by $\varkappa \circ \eta: Q \rightarrow R$ by 3.4. Proceeding as above, we obtain:

$$
T_{\mathfrak{m}^{j}}^{R}(z)=T_{\mathfrak{m}^{j}}^{Q}(z) \cdot \frac{\mathrm{P}_{k}^{R}(z)}{\mathrm{P}_{k}^{Q}(z)}=\frac{T_{\mathfrak{m}^{j}}^{Q}(z)}{d_{R}(z)}
$$

where the second equality is obtained using $3.3(4)$.

In $\left[13\right.$, Lemma 4.4] it is proved that the map $\operatorname{Tor}_{i}^{Q}\left(\nu_{r+1}, k\right)$ is zero for all $i \neq e$ and is bijective for $i=e$. The argument given in the proof there, with a minor adjustment, shows that the following more general statement holds: For any $j$ with $r \leq j \leq s$, the map $\operatorname{Tor}_{i}^{Q}\left(\nu_{j+1}, k\right)$ is zero for all $i \neq e$ and is bijective for $i=e$.

Note that $\operatorname{Tor}_{e}^{Q}\left(\mathfrak{m}^{j}, k\right) \cong \operatorname{Soc}\left(\mathfrak{m}^{j}\right)$, the socle of $\mathfrak{m}^{j}$. Since $R$ is Gorenstein, $\operatorname{rank}_{k} \operatorname{Soc}\left(\mathfrak{m}^{j}\right)=\operatorname{rank}_{k} \operatorname{Soc}(R)=1$. It follows that

$$
T_{\mathfrak{m}^{j}}^{Q}(z)=z^{e}
$$

and thus $T_{\mathbf{m}^{j}}^{R}(z)$ is a quotient of a polynomial of degree $e$ by a polynomial of degree $e+2$. We conclude that $T_{\mathfrak{m}^{j}}^{R}(z)$ is not a polynomial and thus $\operatorname{Tor}_{i}^{R}\left(\nu_{j+1}, k\right) \neq 0$ for infinitely many $i$. (On the other hand, note that $T_{\mathfrak{m}^{j}}^{R}(z)$ is a multiple of $z^{e}$ in $\mathbb{Z}[[z]]$, and this implies that $\operatorname{Tor}_{i}^{R}\left(\nu_{j+1}, k\right)=0$ for all $i<e$. $)$ 
Case 3. Assume $j=t-1$ and $j<r$. Since $r=t-1$ when $s$ is even, this case can happen only when $s$ is odd. In this case, one has $r=t$, hence $j=r-1$ as well. In particular, $j+1=r$ and we use the already established fact that $T_{\mathfrak{m}^{r}}^{R}(z)=0$ in the second line below, in order to replace $\mathrm{P}_{\mathfrak{m}^{j+1}}^{R}(z)$.

$$
\begin{aligned}
z(1+z) T_{\mathfrak{m}^{j}}^{R}(z) & =z \mathrm{P}_{\mathfrak{m}^{j}}^{R}(z)-a_{j} z \mathrm{P}_{k}^{R}(z)+z^{2} \mathrm{P}_{\mathfrak{m}^{j+1}}^{R}(z) \\
& =\left(\mathrm{P}_{R / \mathfrak{m}^{j}}^{R}(z)-1\right)-a_{j} z \mathrm{P}_{k}^{R}(z)+z^{2}\left(a_{j+1} \mathrm{P}_{k}^{R}(z)-z \mathrm{P}_{\mathfrak{m}^{j+2}}^{R}(z)\right) \\
& =\left(\mathrm{P}_{R / \mathfrak{m}^{j}}^{Q}(z)-\left(a_{j} z-a_{j+1} z^{2}\right) \mathrm{P}_{k}^{Q}(z)-z^{3} \mathrm{P}_{\mathfrak{m}^{j+2}}^{Q}(z)\right) \cdot \frac{\mathrm{P}_{k}^{R}(z)}{\mathrm{P}_{k}^{Q}(z)}-1
\end{aligned}
$$

For the last equality, we have used the definition of inertness and the fact that the $R$-modules $R / \mathfrak{m}^{j}, \mathfrak{m}^{j+2}$ and $k$ are all inert by $\varkappa \circ \eta$; this can be seen using again 3.4 , since $j=t-1$ and $j+2=r+1$. Using 3.3(4), we have

$$
T_{\mathfrak{m}^{j}}^{R}(z)=\frac{\mathrm{P}_{R / \mathfrak{m}^{j}}^{Q}(z)-\left(a_{j} z-a_{j+1} z^{2}\right) \mathrm{P}_{k}^{Q}(z)-z^{3} \mathrm{P}_{\mathfrak{m}^{j+2}}^{Q}(z)-d_{R}(z)}{z(z+1) d_{R}(z)}
$$

Since $d_{R}(z)$ has degree $e+2$ and $\mathrm{P}_{\mathfrak{m}^{j+2}}^{Q}(z)$ is a polynomial of degree $e$ (note that $\mathfrak{m}^{j+2}=\mathfrak{m}^{t+1} \neq 0$ ), the outcome of this computation is that $T_{\mathfrak{m}^{j}}^{R}(z)$ is a quotient of a polynomial of degree $e+3$ by a polynomial of degree $e+4$. Again, it is clear that $T_{\mathfrak{m}^{j}}^{R}(z)$ cannot be a polynomial.

Case 4. Assume $j \leq t-2$. We have:

$$
\begin{aligned}
z(1+z) T_{\mathfrak{m}^{j}}^{R}(z) & =z \mathrm{P}_{\mathfrak{m}^{j}}^{R}(z)-a_{j} z \mathrm{P}_{k}^{R}(z)+z^{2} \mathrm{P}_{\mathfrak{m}^{j+1}}^{R}(z) \\
& =\left(\mathrm{P}_{R / \mathfrak{m}^{j}}^{R}(z)-1\right)-a_{j} z \mathrm{P}_{k}^{R}(z)+z\left(\mathrm{P}_{R / \mathfrak{m}^{j+1}}^{R}(z)-1\right) \\
& =\left(\mathrm{P}_{R / \mathfrak{m}^{j}}^{Q}(z)-a_{j} z \mathrm{P}_{k}^{Q}(z)+z \mathrm{P}_{R / \mathfrak{m}^{j+1}}^{Q}(z)\right) \cdot \frac{\mathrm{P}_{k}^{R}(z)}{\mathrm{P}_{k}^{Q}(z)}-1-z
\end{aligned}
$$

where the third equality is due to the fact that $R / \mathfrak{m}^{j}, R / \mathfrak{m}^{j+1}$ and $k$ are all inert by $\varkappa \circ \eta$, in view of 3.4. Using 3.3(4) as above, one sees that $T_{\mathrm{m}^{j}}^{R}(z)$ can be written as a qotient of a polynomial of degree $e+3$ by a polynomial of degree $e+4$, and thus it is not a polynomial.

Since $\operatorname{Tor}_{*}^{R}\left(\nu_{j}, k\right)=0$ is clearly zero when $j>s$, we exhausted all cases for $j$.

\section{Acknowledgement}

We would like to thank Frank Moore for a useful discussion about $\mathcal{K}_{2}$ algebras, and Luchezar Avramov and the referee for suggestions regarding the exposition.

\section{REFERENCES}

[1] L. L. Avramov, Infinite Free Resolutions, Six lectures on commutative algebra, (Bellaterra, 1996), Progr. in Math. 166, Birkhäuser, Basel, 1998; 1-118.

[2] L. L. Avramov, Small homomorphisms of local rings, J. Algebra 50 (1978), 400-453.

[3] M. Boij, Betti numbers of compressed level algebras, J. Pure Appl. Algebra 134 (1999), $111-131$.

[4] T. Cassidy, B. Shelton, Generalizing the notion of Koszul algebra, Math. Z. 260 (2008), no. 1, 93-114.

[5] E. S. Golod, On the homologies of certain local rings, Soviet Math. Dokl. 3 (1962), 745-748; translated from Dokl. Akad. Nauk. SSSR 144 (1962), 479-482 [Russian].

[6] A. Iarrobino, Compressed algebras: Artin algebras having given socle degrees and maximal length, Trans. Amer. Math. Soc. 285 (1984), 337-378. 
J. HOFFMEIER AND L. M. ŞEGA

[7] J. Lescot, Séries de Poincaré et modules inertes, J. Algebra 132 (1990), 22-49.

[8] G. Levin, Finitely generated Ext algebras, Math. Scand. 49 (1981), 161-180.

[9] G. Levin, Local rings and Golod homomorphisms, J. Algebra 37 (1975), 266-289.

[10] G. Levin, Modules and Golod homomorphisms, J. Pure Appl. Algebra 38 (1985), 299-304.

[11] C. Löfwall, On the subalgebra generated by the one-dimensional elements in the Yoneda Extalgebra, Algebra, algebraic topology and their interactions (Stockholm, 1983), Lecture Notes in Math., 1183, Springer, Berlin, 1986; 291-338.

[12] J.-E. Ross, Relations between Poincar-Betti series of loop spaces and of local rings, Séminaire d'Algèbre Paul Dubreil 31ème année (Paris, 1977-1978), pp. 285-322, Lecture Notes in Math., 740, Springer, Berlin, 1979.

[13] M. E. Rossi, L. M. Şega, Poincaré series of modules over compressed Gorenstein local rings, Adv. Math. 259 (2014), 421-447.

[14] L. M. Şega, Homological properties of powers of the maximal ideal of a local ring, J. Algebra 241 (2001), 827-858.

[15] L. M. Şega, On the linearity defect of the residue field, J. Algebra 384 (2013), 276-290.

[16] G. Sjödin, A set of generators for $\operatorname{Ext}_{R}(k, k)$. Math. Scand. 38 (1976), no. 2, 199-210.

Justin Hoffmeier, Department of Mathematics and Statistics, Northwest Missouri State University, Maryville, MO 64468, U.S.A.

E-mail address: jhoff@nwmissouri.edu

Liana M. Şega, Department of Mathematics and Statistics, University of Missouri, KANSAS City, MO 64110, U.S.A.

E-mail address: segal@umkc.edu 